\title{
ZUR ERINNERUNG
}

\author{
AN \\ AXEL HARNACK
}

VON

Dr. A. vOSS,

ORD. PROFESSOR DKR MATHEMATTK

AN DER TECHXISCHEN HOCHSCHULE ZU MÜNCHEN.

DRUCK VON B. G. TEUBNER IN LEIPZIG.

1888. 


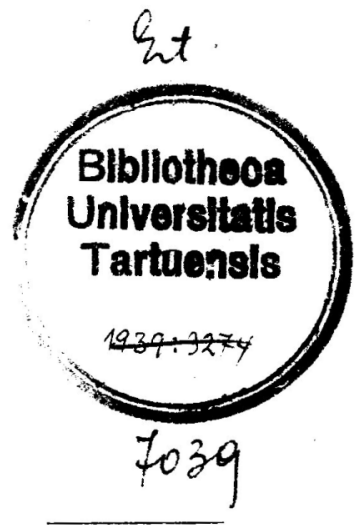

Separatabzug aus dem 32. Bande der Mathematischen Annalen. 


\section{Zur Erinnerang an Axel Harnack.}

Von

A. Voss in Múnchen.

Der am 3. April dieses Jahres als Professor der Mathematik am k. Polytechnikum in Dresden verstorbene Axel Harnack gehört zu jenen Männern, die sich durch Vielseitigkeit der Begabung nnd Reichthum der Gedanken in hervorragender Weise ausgezeichnet haben. In der ersten Hälfte des kurzen, kaum mehr als zwölf Jahre umfassenden Zeitraums, während dessen es ihm vergönnt war, selbständig zu arbeiten, mit geometrischen Untersuchungen beschäftigt, zu deren Behandlung gerade in derjenigen Richtung, wie sie $\mathrm{Clebsch}$ in so charakteristischer Weise vertreten hat, ihn ein besonders glückliches Talent befähigte, hat er sich in den letzten Jahren seines Lebens fast ausschliesslich und mit immer steigendem Erfolge jenen grossen Fragen der reinen Analysis zugewandt, die seit Dirichlet's Arbeiten über die Darstellung willkürlicher Functionen fortwährend neuen Anstoss zu tieferem Eindringen in die Fundamentalbegriffe der Lehre von den Functionen gegeben haben.

Carl Gustav Axel Harnack ward geboren am 7. Mai $1851 \mathrm{zu}$ Dorpat als Sohn des ausgezeichneten Theologen Theodosius Harnack, jetzt Prof. emer. daselbst. Schon in früher Jugend entwickelte sich bei ihm eine lebhafte Neigung für mathematische und physicalische Gegenstände; die entscheidende Anregung, sich ganz der Mathematik zu widmen, verdankte er auf der Universität Dorpat vorzugsweise seinen Lehrern A. v. Oettingen und F، Minding. Nach Vollendung seiner Studien, von deren Erfolg unter anderen auch seine von der Dorpater physical. medicin. Facultät mit dem ersten Preise gekrönte Arbeit*) "Ueber Maxima und Minima von Ellipseninhalten in Kegelschnittreihen und Netzen" zeugt, wandte er sich 1873 nach Deutschland, wo neben worden.

*) Diese bereits 1872 vollendete Arbeit ist indess nicht veröffentlicht 
den analytischen Disciplinen gerade zu jener Zeit durch die eminente Begabung, mit der Clebsch seinen Schülern die Arbeiten der deutschen und englischen Geometer zugänglich zu machen wusste, auch das Interesse für geometrische Studien weitergehender Art sich in besonders lebhafter Entwickelung befand. In Erlangen, wo sein Vater 1853-1866 als Professor gewirkt, er selbst einen Theil seiner Jugendzeit verlebt hatte, zog er bald die Aufmerksamkeit Klein's. auf sich. Vor allen interessirte ihn der Kreis von geometrischen Fragen, welche sich an die von Clebsch und Gordan bearbeitete Theorie der Abel'schen Functionen angeschlossen hatten, in die er namentlich durch Klein eingeführt wurde, sowie die Fortbildung, welche die Algebra in der Bewältigung der auf die Theorie der homogenen Functionen gerichteten Aufgaben erfahren hatte, und in der er sich der ausgezeichneten Förderung Gordan's erfreuen durfte, der nicht lange darnach von Giessen nach Erlangen berufen war. Durch Klein's Anregung entstand zunächst seine Inauguraldissertation*) „Ueber die Verwerthung der elliptischen Functionen für die Geometrie der Curven dritter Ordnung", in welcher zum ersten Mal der Gedanke ausgeführt ist, die von Clebsch begründete Parameterdarstellung der Curve dritter Ordnung nach den von Klein eingeführten Gesichtspunkten**) zu entwickeln und durch die mannigfachen Beziehungen, welche die von Clebsch begonnene Connextheorie ${ }^{* * *}$ ) bietet, zu bereichern. Eine weitere Ausdehnung namentlich nach dieser letzteren und der formentheoretischen Seite hin gab er sodann diesen Untersuchungen in der Arbeit über cubische ternäre Formen. $\dagger$ )

Mit diesen ersten Arbeiten stehen in enger Verbindung seine Note über den Abel'schen Satz $\dagger$ ), dem er eine neue und für geometrische Anwendungen elegante Form abzugewinnen wusste, ferner seine Untersuchungen über die Behandlung der algebraischen Differentiale in homogenen Coordinaten, $+f \dagger$ ) die fast gleichzeitig auch von Lindemann in den von letzterem bearbeiteten Vorlesungen von Clebsch entscheidend gefördert ist, sowie der von Harnack zuerst aus-

*) Inauguraldissertation, Leipzig 1875, auch erschienen Mathem. Annalen Bd. IX, S. 1.

**) Klei.u, Ueber eine neue Art der Riemann'schen Flächen, Math. Ann. Bd. VII, S. 558 .

***) Vgl. die Darlegungen von Clebsch in den Math. Ann. und den von Lindemann bearbeiteten Vorlesungen, S. 924-1037.

†) Zur Theorie der ternären cubischen Formen, Math. Ann. Bd. IX, S. 218.

††) Berichte der phys. med. Societät zu Erlangen, 1875; dann auch Math. Ann. Bd. IX, S. 383.

it) Ueber eine Behandlungsweise der algebraischen Differentiale in homogenen Coordinaten, Math. Ann. Bd. IX, S. 371. Man vergleiche auch die Lindemann'sche Arbeit in den Vorlesungen von Clebsch, S. 764-923. 
gesprochene Beweis des Satzes, dass eine ebene Curve vom Geschlechte $p$ höchstens aus $p+1$ getrennten Zügen bestehen kann. Wenn gleich dieser Satz auch auf anderen Wegen gewonnen werden kann,*) so wird doch die völlig elementare Art, in der Harnack, auf Vorstellungen von Möbius und Plücker zurückgehend, die Existenz von Curven der n. Ordnung, denen die genannte Eigenschaft. zukommt, begründete, von dauerndem Werthe bleiben.

Noch während des Entstehens dieser Arbeiten hatte sich Harnack als Docent für Mathematik an der Universität Leipzig habilitirt (Herbst 1875).**) Er hat hier vorzugsweise über geometrische Fragen gelesen, auf die er sich sowohl durch die Richtung, die seine eigenen Studien genommen, als auch durch die ihm übertragene Herausgabe der Hankel'schen Vorlesungen über synthetische Geometrie hingewiesen sah.**) Seine ausgezeichnete Lehrbegabung, das ihm eigene Geschick, bei sorgfältiger algebraischer Behandlung des einzelnen stets den Blick auf principielle Fragen zu concentriren, gewannen ihm gleich von Anfang an einen zahlreichen Kreis von Zuhörern und die Anerkennung der Professoren Scheibner und Neumann, mit denen er dauernd in pietätvoller Beziehung geblieben ist.

Die so glücklich begonnene Leipziger Thätigkeit musste Harnack sich gleichwohl entschliessen, aufzugeben, als er im Herbst 1876 an die technische Hochschule zu Darmstadt als Professor der Mathematik berufen wurde. Ostern 1877 begründete er daselbst seinen eigenen Hausstand mit Elisabeth von Oettingen aus Ludenhof bei Dorpat. Nur kurze Zeit hat er in Darmstadt gewirkt; schon im Herbst 1877 siedelte er nach Dresden über.

Das Polytechnicum zu Dresden hatte unter Zeuner's Direction einen mächtigen Aufschwung genommen. Derselbe eröffnete insbesondere auch den mathematischen Studien eine weitere Perspective, die unter S c hlö mil ch's ausgezeichneter Thätigkeit daselbst gepflegt waren,

*) Ueber die Vieltheiligkeit der ebenen algebraischen Curven, Math. Ann. Bd. X, S. 189. Einen anderen, auf der Betrachtung der symmetrischen Riemann'schen Flächen beruhenden Beweis des Harnack'schen Satzes gab Klein, Rie-' mann's Theorie der algebraischen Functionen, Leipzig 1882, S. 72. Uebrigens findet sich auch schon bei Schottky, (conforme Abbildung mehrfach zusammenhängender ebener Flächen, Journal v. Crelle 83 (1877)) der Nachweis der Existenz von ebenen Curven des Geschlechtes $\rho$ mit $\rho+1$ geschlossenen Theilen, daselbst $\S 5$, S. 314 .

**) Als Habilitationsschrift hatte Harnack die, Anmerk. S. 4 ††, erwähnte Arbeit eingereicht; den Gegenstand seiner Habilitationsvorlesung bildete eine geschichtliche Darlegung des Begriffs der algebraischen Curven, in der zum Schluss auch der soeben angegebene Satz berührt wird.

****) Hermann Hankel, Die Elemente der projectivischen Geometrie in synthetischer Behandlung, herausgegeben von Harnack, Leipzig 1875, Teubner. 
und für die nach dessen Uebergang in einen anderen Wirkungskreis in Königsberger aufs neue eine gefeierte Kraft gewonnen war. Nicht ohne Zagen nahm Harnack den Ruf an, der ihm bei Königs berger's Berufung nach Wien zu Theil wurde; mit Eifer aber suchte er den vielfachen Pflichten, welche die Organisation der Anstalt von ihm forderte, gerecht zu werden.

Die Aufgabe, ein grösseres Publicum in die Principien der Differential- und Integralrechnung mit einer auch für weitergehende rein wissenschaftliche Bedürfnisse ausreichenden Allgemeinheit einzuführen, nahm fortan sein ganżes Interesse in Anspruch. Wie er selbst über seine Aufgabe als Lehrer an einer technischen Hochschule dachte, hat er aufs klarste gelegentlich einer Recension im Civilingenieur ausgesprochen.*) ,Sieht man von der elementaren Mathematik ab, so erhebt sich immer-wieder das schwierige Problem, welches bei allen Lehrbüchern der sogenannten höheren Mathematik, ja beì dem gesammten mathematischen Studium, zumal an den technischen Hochschulen sich geltend macht: die Frage nach der Behandlungsweise der einzelnen Disciplinen hinsichtlich der Vollständigkeit und Präcision. Letztere zumal erfordert nach dem gegenwärtigen Stande der Wissenschaft eine so grosse Vertiefung, dass man von vornherein darauf verzichten muss, ihr überall dadurch gerecht zu werden, dass man die einzelnen Theoreme in ihrem vollen Umfange klar legt, wohl aber lässt sie sich durch eine genaue Einschränkung derselben jeder Zeit aufrecht erhalten. Die Aufgabe, welche der mathematische Unterricht und jedes Lehrbuch zu lösen haben, ist also meiner Meinung nach so zu fixiren: klare und vollständige Auseinandersetzung der grundlegenden Begriffe, möglichste Beschränkung der reinen Theorie nebst scharfer Formulirung der Lehrsätze innerhalb gegẹbener eng gezogener Voraussetzungen, Reichhaltigkeit in der Anwendung auf gebotene Probléme.“

Aus diesen Vorträgen ist zunächst sein Werk über die Elemente der Differential- und Integralrechnung, herrorgegangen, ${ }^{* *}$ ) in dem er sich allerdings auch viel weitergehende Aufgaben gestellt hatte. Dasselbe hat wegen der geschickten und originellen Darstellung, in der die principiellen Fragen überall an die Spitze treten, ohne dass doch die Anwendung auf das einzelne vernachlässigt wird, und die Betrachtung sich in völlig abstracte dem Verständnisse der Studirenden weniger

*) Das Citat im Texte ist entnommen aus der von Harnack verfassten Anzeige des von Heger und Reidt veröffentlichten Handbuches der Mathematik, Breslan 1879 und 1881, Civilingenieur Bd. XXIX.

**) Die Elemente der Differential- und Integralrechnung zur Einführung in das Studium dargestellt von Axel Harnack, mit Figuren im Text, Leipzig 1881, Teubner. 
zugängliche Fragen verliert, allseitigen Beifall gefunden.*) Sein Wunsch, dasselbe bei Gelegenheit einer neuen Auflage zu einer theoretischen Darlegung der Grundprincipien im Sinne der neueren Functionentheorie zu gestalten, dagegen die Erörterung der mehr elementaren Anwendungen zurücktreten zu lassen, für welche er inzwischen selbst durch die alsbald zu erwähnende Bearbeitung des Serret'schen Cours de calcul différentiel et intégral den angehenden Mathematikern ein vorzügliches Werk geboten hatte, ist leider nicht in Erfüllung gegangen; in seinem Nachlasse**) haben sich übrigens keine Vorarbeiten zu einer solchen Bearbeitung vorgefunden.

Damit beginnt zugleich die zweite Periode in der wissenschaftlichen Thätigkeit Harnack's; in der That hat er seitdem sich fast ausschliesslich analytischen Untersuchungen zugewandt. Zunächst nahm er die Bemerkung Töpler's, ${ }^{* * *}$ ) dass die Coefficienten der Fourier'schen Reihe sich vermittelst einer der Methode der kleinsten Quadrate nachgebildeten Betrachtung ergeben, zum Ausgangspunkt von Untersuchungen über die Fourier'sche Reihe. Bekanntlich hat Du-BoisReymond $\dagger$ ) zuerst den Beweis des wichtigen Satzes gegeben, dass die Coefficienten einer trigonometrischen Reihe in die Fourier'sche Form gebracht werden können, wenn die Function endlich und integrabel ist, oder in den Punkten einer Menge erster Gattung unendlich wird. Harnack's Bestreben war zunächst darauf gerichtet, diese Untersuchungen wo möglich zu vereinfachen und zu erweitern. Indem er die allerdings beschränkende Voraussetzung einführte $\dagger \dagger$ ), dass die dargestellte Function nebst ihrem Quadrate integrabel sei, liess sich nicht allein das schliessliche Verschwinden der Fourier'schen Coefficienten sondern auch die im allgemeinen gleichmässige Convergenz des beliebig weit ausgedehnten Restgliedes der Reihe nachweisen. Reicht auch die angegebene Voraussetzung nicht aus, um die gliedweise Integrirbarkeit der Reihe zu erweisen, 'so scheinep doch diose

*) Man sehe die Recensionen von H. Weber, Schlömilch's Zeitschrift für Math. u. Physik, Bd. 27, S. 161; Wangerin, Deutsche Literaturzeitung vom Jahre 1882; Hoppe, Fortschritte der Mathematik für das Jahr 1881, S. 202; Godt, Zeitschrift für math. u. naturw. Unterricht von Hoffmann, 1883, S. 51; Günther, Blätter für das bayerische Gymnasialwesen 1882, S. 165.

**) Ausser den bereits veröffentlichten Schriften hat sich im Nachlasse Harnack's noch die sorgfältig ausgeführte Habilitationsvorlesung, sowie ein allerdings nur zum Theil fertiges Manuscript vorgefunden, über das S. 13, Anm. berichtet ist.

***) Anzeiger d. Akad. zu Wien, 7. Dec. 1876; Repertorium d. Math. Bd. I, S. 402.

Seite 119.

†) Abhandlungen der k. bayerischen Academie, II. Cl., Bd. XII, 1. Abth.

††) Math. Ann. Bd. XVII, S. 123; Bd. XIX, S. 235 u. 324. 
Betrachtungen geeignet, auch weitergehende Untersuchungen, wie sie Harnack namentlich in seiner Schrift de la Série de Fourier, Paris Gauthier-Villars $1883,{ }^{*}$ ) ausgeführt hat, zu fördern. Auch von anderen Mathematikern, z. B. Halphen**), der unabhängig davon denselben Gang eingeschlagen hatte, ist die bereits von Harnack erkannte Verwendbarkeit für analoge Fragen betont worden, obwohl andererseits kein Zweifel darüber bestehen kann, dass die weitere Verfolgung des von $\mathrm{Da}$-Bois Reymond eingeschlagenen höchst scharfsinnigen Weges, wie sie von $H$ Ḧlder ausgeführt ist, zu allgemeineren Resultaten hinleitet. ***)

In engem Zusammenhange steht hiermit die Einführung des Begriffes der discreten Punktmenge, mit dem Harnack an die von Hankel in dessen Tübinger Festschrift $\dagger$ ) ausgesprochenen Ideen anknüpfte. Eine Punktmenge heisst darnach discret, ††) wenn sich sämmtliche Punkte derselben in eine endliche Anzahl von Intervallen einschliessen lassen, deren Summe beliebig klein gemacht werden kann, mag dabei auch die Anzahl jener Intervalle über jeden Betrag wachsen.

Berührte sich einerseits diese Begriffsbildung mit den tiefgehenden und wichtigen Untersuchungen G. Cantor's über Punktmengen überhaupt, so steht doch Harnack mit derselben zunächst auf den dem Riemann'schen Integralbegriff erwachsenen Gesichtspunkten. In der That hat aueh die discrete Menge bei den Problemen der Integralrechnung eine wesentliche Wichtigkeit, während für die fundamentalen Untersuchungen, die gleichzeitig die Differential- und Integralrechnung betreffen, die Cantor'schen weit allgemeineren Conceptionen massgebend erscheinen, wie dies z. B. auch aus den Scheeffer'schen Arbeiten $\dagger_{\dagger} \dagger$ ) hervorgeht.

Die Untersuchungen über die Fourier'sche Reihe lenkten

*) Diese Schrift findet sich auch im Darboux'schen Bulletin des sciences math. et. astr. 1883 . Ser. II, t. 6.

**) Vgl. Halphen, Sur la série de Fourier, Comptes Rendus t. 95, S. 1217 und t. 96, S. 168; ebendaselbst auch t. 95, S. 967 die Bemerkung von Hugoniot, Sur le développement des fonctions en séries.

***) Hölder, Zur Theorie der trigonometrischen Reihen, Math. Annalen Bd. XXIV, S. 181.

†) Hankel, Untersuchungen über die unendlich oft oscillirenden und unstetigen Functionen, Festschrift der Universität Tübingen 1870; wieder abgedruckt Math. Ann. Bd. XX, S. 63; vgl. besonders S. 87 u. ff.

H) Siehe Math. Ann. Bd. XXIV, S. 218. Der Begriff der discreten Menge bei Harnack deckt sich übrigens mit dem der Punktmenge vom Inhalt Null bei G. Cantor.

$\dagger_{\dagger}+$ ) Ludwig Scheeffer, Zur Theorie der stetigen Functionen einer reellen Veränderlichen Act. Math. V, S. 183 und 278, ferner allgemeine Untersuchungen über Rectification der Curven, daselbst S. 52. Es ist dies übrigens auch von Harnack selbst bemerkt worden, z. B. Math. Ann. Bd. XXIV, S. 231, Anmerk. 
Harnack's Interesse weiter auf den Fundamentalsatz aus der Theorie der Functionen einer complexen Variabelen, nach welchem jede endliche eindeutige und stetige Function, welche in einem Gebiete eine endliche und stetige Ableitung besitzt, in demselben durch eine Potenzreihe dargestellt werden kann. Er zeigte hier,*) wie dieser Satz nebst der Laurent'schen Erweiterung sich unmittelbar aus der Theorie der Fourier'schen Reihe gewinnen lässt, und wie die von Riemann gegebenen Unstetigkeitsbedingungen präcisirt werden können.

Doch schon während dieser Zeit machten sich die Anfänge des Leidens bemerkbar, das auf sein inzwischen auf das glücklichste entwickeltes Familienleben sobald einen trüben Schatten werfen sollte. Von Hause aus zart organisirt, hatte Harnack sich beim Besuche eines Seebades, wie es schien, eine Erkältung zugezogen, die bald einen ernsteren Charakter annahm. Als auch ein Aufenthalt in Botzen, den er im Frühjahr 1883 nahm, keine wesentliche Besserung herbeiführte, war es leider nicht mehr zu verkennen, dass ein tieferes Leiden seinen Organismus ergriffen hatte. Er musste sich entschliessen - wozu ihm die k. Sächs. Regierung in der entgegenkommendsten Weise die Möglichkeit eröffnete, - seine Lehrthätigkeit zeitweilig ganz aufzugeben, um in Davos, wohin ihm seine Familie Ostern 1884 folgte, Kräftigung zu suchen.

Aber auch in dieser Zeit, die sein lebhaftes Pflichtgefühl besonders schwer empfand, ist er nicht müssig gewesen. Nicht nur nahm er auch aus der Ferne lebhaften Antheil an allen Fragen, welche die Dresdner Hochschule berührten; mit staunenswerther Energie erledigte er während des $1 \frac{1}{2}$ jährigen Aufenthaltes in Davos, fern von allen literarischen Hülfsmitteln, die Aufgabe, die er sich in der deutschen Bearbeitung von Serret's Calcul différentiel et intégral **) gestellt hatte. Von dem Werke Serret's hat Harnack eine vorzügliche Uebersetzung geliefert, die soweit wie möglich das Original wiedergiebt; aber in einer Reihe von Zusätzen, die durch kleineren Druck kenntlich gemacht sind, und namentlich in den beiden letzten Bänden dieses grossen Lehrbuches einen bedeutenderen Umfang einnehmen, hat er eine Fülle wichtiger Ergänzungen hinzugefügt, durch welche dasselbe ganz wesentlich gewonnen hat. Namentlich möge hier die musterhafte Darlegung der Theorie der Fourier'schen Reihen und Integrale ${ }^{* * *}$ ) angeführt werden; sie ist geeignet, den angehenden

*) Anwendung der Fourier'schen Reihe auf die Theorie der Functionen einer complexen Veränderlichen, Math. Ann. Bd...XXI, S. 305.

**) Serret, Cours de calcul différential et intégral, II édition, Paris, Gauthier-Villars 1880.

***) Siehe in Serret-Harnack, Lehrbuch der Differentialrechnung etc. die Abschnitte: Grundriss der Theorie der Fourier'schen Reihe und der Fourier'schen 
Mathematikern auch schon in den ersten Semestern den Zugang zu den Untersuchungen Riemann's und anderer Forscher zu eröffnen.

Als Harnack Ostern 1885 zurückkehrte, schien seine Gesundheit gekräftigt, wenngleich er sich selbst keineswegs verhehlte, dass sein eigentliches Leiden nicht gehoben war. Aber seine Arbeitskraft hatte sich ungeschwächt erhalten; ja, sie schien noch gewachsen.

Die Untersuchungen über die Darstellung willkürlicher Functionen einer reellen Variabelen mussten Harnack naturgemäss auf die erweiterten Probleme führen, welche mit dem sogenannten Dirichlet'schen Princip aufgetreten sind. Bekanntlich ist der Beweis des Satzes, dass die Differentialgleichung $\Delta^{2} u=0$ stets ein Integral besitzt, welches in der Begrenzung eines ebenen oder räumlichen Gebietes vorgeschriebene Werthe annimmt, in der Weise, wie derselbe, von W. Thomson zuerst ausgesprochen, namentlich seit Riemann für die Functionentheorie eine hervorragende Wic̈htigkeit erlangt hat, längst als nicht ausreichend erkannt worden. Es ist das Verdienst von Neumann und H. A. Schwarz*), diese Schwierigkeit zuerst unter bestimmten Voraussetzungen überwunden zu haben. In der Programmschrift der eidgen. polytechn. Schule von 1870 zeigte Schwarz, wie aus der conformen Abbildung eines Polygons auf die Halbebene die Existenz der Green'schen Function mittels bestimmter Convergenzprocesse erkannt werden kann, falls das Polygon in eine nach Aussen überall convexe Curve übergeht. Einen anderen Beweis, der zum Theil auf erweiterten Voraussetzungen beruht; hat derselbe in den Berichten der Berliner Academie gegeben (1870). Zu derselben Zeit gelang es Neumann,*) den genanuten Satz sowohl für die Ebene als den Raum zu beweisen, sobald die Begrenzung überall convex nach aussen ist, und mit Hülfe einer gleichzeitig von Schwarz gefundenen Methode auch auf anderweitig begrenzte, auch mehrfach zusammenhängende, Gebiete zu erweitern. Harnack stellte sich nun die Aufgabe, diese Resultate auch für den Fall, dass die Berandung, die nicht nur eine analytische Curve zu sein braucht, von diesen Einschränkungen befreit ist, directer zu gewinnen. In der That zeigte er, in einer der K. Sächs. Gesellschaft der Wissenschaften am 2. Mai 1886 übergebenen Abhandlung**), wie der von $\operatorname{Sch}$ warz eingeschlagene

Integrale, Bd. II. S. 343-380; ferner: Zur Integration der partiellen Differentialgleichungen in der Theorie der Functionen einer complexen Veränderlichen, Bd. III. S. 378-388, sowie auch die einschlägige Darstellung in den "Grundlagen der Theorie des logarithmischen Potentials". Leipzig 1887.

*) Vgl. die Darlegung bei Klein, Math. Ann. Bd. XXI, S. 153, Literarisches zum Dirichlet'schen Princip.

**) Existenzbeweise zur Theorie des Potentiales in der Ebene und im Raume, Berichte d. sächs. Gesellschaft d. W. 2. Mai 1886. 
Weg sowohl in der Ebene als auch im Raume, wenigstens für ein einfach zusammenhängendes Gebiet durchgeführt werden kann. Die voll-, ständige Darlegung dieser Untersuchungen, namentlich für den Raum war ihm nicht mehr beschieden.

Dagegen hat er in seinem letzten grösseren Werke, über die Theorie des Potentiales*) für die Ebene eine zusammenfassende Darstellung der Schwarz'schen und Neumann'schen Untersuchungen in Verbindung mit seinen eigenen Resultaten gegeben, welche zugleich die wesentlichsten Gesichtspunkte enthält, die er bei dem Problem im Raume einzuhalten beabsichtigte.

Von Ostern 1885 an hatte Harnack seine Vorlesungen ohne Unterbrechung wieder aufgenommen, obwohl sein Befinden ein wechselndes war und namentlich im Sommer 1887 seine Angehörigen wieder mit Sorge erfüllen musste. Im Winter darauf kam wieder eine günstigere Periode für ihn; noch im December nahm er Theil an einer Sitzung der Leipziger Gesellschaft der Wissenschaften, zu deren ordentlichen Mitgliedern er seit 1886 gehörte, ja er unternahm selbst kleinere Reisen, um seinen fast ganz unterbrochenen persönlichen Verkehr mit Fachgenossen zu erweitern. Aber schon im Februar sah er sich wieder gezwungen, seine Ausgänge auf das nothwendigste Mass zu beschränken. Noch hoffte er neue Kräftigung in der milden Luft der oberitalienischen Seen zu finden; mit ungewöhnlicher Energie führte er seine Vorlesungen weiter, um ohne mit seinem eigenen Pflichtgefühl in Widerstreit zu kommen, in den Osterferien eine Erholungsreise etwas länger ausdehnen zu können. Es sollte nicht sein. Am 16. März brach er mitten in der Vorlesung zusammen; sein Leiden, das bisher doch nur langsame Fortschritte gemacht $\mathrm{zu}$ haben schien, nahm plötzlich eine unerwartete unheilvolle Wendung: schon nach wenigen Tagen ward er den Seinen entrissen. Die Vorboten dieser Erkrankung waren schon eingetreten, als er sein letztes Manuscript**) an die Redaction dieser Annalen einsandte, und selbst in den letzten Tagen beschäftigte ihn noch der Gedanke an eine Revision seiner Elemente der Differentialrechnung für eine englische Uebersetzung, zu der er seine Genehmigung ertheilt hatte.

Axel Harnack besass eine Persönlichkeit, die auf alle, die mit ihm in Berührung gekommen sind, vom ersten Augenblick an durch die unmittelbar hervortretende Reinheit und Liebenswürdigkeit ihres Charakters einen tiefen Eindruck machte. Liebevoll und kindlich ein-

*) Grundlagen der Theorie des logarithmischen Potentials und der Potentialfunctionen in der Ebene, Leipzig 1887.

**) Es ist dies die hier anschliessend veröffentlichte Schrift Harnack's über Cauchy's zweiten Beweis für die Convergenz der Fourier'schen Reihe. 
fach im häuslichen Kreise, voll von sittlichem Ernste und doch heiterer Fröhlichkeit im Verkehr mit seinen Collegen und Freunden, erfüllt von dem idealsten wissenschaftlichen Streben und dabei doch wieder mit sicherer Festigkeit die gegebenen Verhältnisse unverückt im Auge behaltend, übte er nicht nur auf seine Schüler einen mächtigen Einfluss, auch ältere Männer ordneten sich gerne dem um so vieles jüngeren unter, ohne dass er in seiner Bescheidenheit den Wunsch dazu hätte hervortreten lassen. Eines hohen Vertrauens erfreute er sich nicht nur unter seinen Collegen, sondern auch in weiteren Kreisen, insbesondere auch in seiner alten Heimat, der er stets die treueste Anhänglichheit bewahrt hat. Die Macht der Rede stand ihm in seltenem Masse zu Gebote; mühelos gelang es ihm, den Reichthum seiner Gedanken zugleich in die treffendste Form zu kleiden. Bedeutend war seine Begabung für organisatorische Fragen; bei schwierigen Angelegenheiten wusste er immer den Nagel auf den Kopf zu treffen. Sein fruchtbares Wirken als Lehrer fand auch vielfache äussere Anerkennung: in Dorpat dachte man daran ihn als Nachfolger Minding's zu gewinnen; 1877 erhielt er einen Ruf an die Universität Rostock, 1882 an die technische Hochschule zu Aachen. Beidemale glaubte er ablehnen zu sollen; einem 1883. an ihn ergangenen Rufe an die technische Hochschule zu München war er bereit zu folgen, doch trat seine Gesundheit hindernd dazwischen.

Harnack war zugleich ausgezeichnet durch eine vorzügliche literarische und ästhetische Bildung, die er gern in der Geselligkeit des Hauses weiter förderte; geschichtliche und philosophische Studien beschäftigten ihn unausgesetzt in seinen Mussestunden. In die Einsamkeit von Davos begleitete ihn das zu eben der Zeit vollendete grosse Werk von Wundt*) über die Principien der Erkenntniss, und die Art, wie er sich im eigenen Denken über die Grundlagen aller Naturerkenntniss klar zu werden suchte, mag der seinem Freunde und Lehrer A. v. 0 ettingen gewidmete Vortrag bezeichnen, den er, von dort zuräckgekehrt, in der naturwissenschaftlichen Gesellschaft zu Dresden hielt.**) Leibniz' grossartige Persönlichkeit fesselte ihn begreiflicherweise insbesondere. Von Interesse wird immer die kurze und dabei doch so lebendige Darstellung von Leibniz' Wirken***) bleiben, welche er Ostern 1877 als Redner zur Geburtstagsfeier seines

*) W. Wundt, Logik. Eine Untersuchung der Principien der Erkenntniss und der Methoden wissenschaftlicher Forschung. Stuttgart, 1880-1883.

**) Naturforschung und Naturphilosophie, Vortrag, gehalten in der naturwissenschaftlichen Gesellschaft zu Dresden von A. Harnack, Leipzig 1885, Teubner.

***) Leibniz' Bedeutung in der Geschichte der Mathematik, Dresden 1887, Zahn und Jaensch. 
Landesherrn gab, und von der man mit M. Cantor*) nur wünschen möchte, dass dieselbe in erweiterter Gestalt hätte veröffentlicht werden können. So erinnert Harnack in mehr als einer Beziehung an den ebenfalls der Wissenschaft so früh entrissenen Hermann Hankel. Beider Arbeiten haben, wie sich leicht auch im einzelnen nachweissen liesse, einen ähnlichen Verlauf genommen; beiden war gemeinsam der feine Sinn für die historische Entwickelung der mathematischen Probleme, der sichere Blick für alle Fragen, die mit den Aufgaben des Unterrichtes zusammenhängen, und das lebendige Interesse für das ihnen anvertraute Lehramt.

Sein Geschick hat die weiteren Erfolge, welche die Wissenschaft seinem beharrlichen Forschen bereit zu halten schien, nicht zur Wirklichkeit werden lassen. Aber in Einem ist er auch in der kurzen Lebenszeit zur Vollendung gereift: in der sittlichen Festigkeit seines Charakters, die, getragen von einer tief religiösen Grundanschauung, welche er als ein Erbtheil seines Vaterhauses doch auch völlig selbstständig in sich entwickelt hatte, die Quelle war, aus der sein den höchsten Aufgaben zugewandtes Streben immer neue Kraft schöpfte. Die Wissenschaft wird ihm dauernd ein ehrenvolles Andenken bewahren. Die einzigartige Persönlichkeit Axel Harnack's aber, in der in harmonischer Weise alles vereinigt war, was den eigentlichen Werth des einzelnen Menschenlebens ausmacht, wird seinen Schülern, seinen Freunden und Fachgenossen stets unvergesslich bleiben.

München, im Mai 1888.

*) Vgl. das Referat von M. Cantor in der Zeitschrift für Math. u. Phys., Bd. 32, S. 321. Dass Harnack auch selbst beabsichtigen mochte, den im Texte angeführten Wunsch M. Cantor's zu erfüllen, scheint aus einer Vorlesung über die geschichtliche Entwickelung der Geometrie insbesondere des 17. Jahrhunderts hervorzugehen, die er im letzten Semester gehalten bat, und die auf selbständigen Studien über diese Periode beruhen dürfte. 


\section{Axel Harnack's literarische Publicationen.}

I.

In den Mathematischen Annalen.

1) Ueber die Verwerthung der elliptischen Functionen für die Geometrie der Curven dritten Grades. Inauguraldissertation, Leipzig 1875; Bd. IX, S. 1.

2) Zur Theorie der ternären cubischen Formen, Bd. IX, S. 218.

3) Ueber eine Behandlungsweise der algebraischen Differentiale in homogenen Coordinaten, Bd. IX, S. 371.

4) Ueber die Vieltheiligkeit der ebenen algebraischen Curven, Bd. X, S. 189.

5) Ueber die Darstellung der Raumcurve vierter Ordnung erster Species und ihres Secantensystems durch doppeltperiodische Functionen, Bd. XII, S. 47.

6) Bemerkungen zur Geometrie auf den Linienflächen vierter Ordnung, Bd. XIII, S. 49.

7) Ueber eine Eigenschaft der Coefficienten der Taylor'schen Reihe, Bd. XIII, S. 555.

8) Notiz über die algebraische Parameterdarstellung der Schnittcurve zweier Flächen zweiter Ordnung, Bd. XV, S. 560 .

9) Ueber die trigonometrische Reihe und die Darstellung willkürlicher Functionen, Bd. XVII, S. 123.

10) Vereinfachung der Beweise in der Theorie der Fourier'schen Reihe, Bd. XIX, S. 235.

11) Berichtigung zu diesem Aufsatze, daselbst S. 521 .

12) Anwendung der Fourier'schen Reihe auf die Theorie der Functionen einer complexen' Veränderlichen, Bd. XXI, S. 305.

13) Die allgemeinen Sätze über den Zusammenhang der Functionen einer reellen Variabelen mit ihren Ableitungen. Erster Theil Bd. XXIII, S. 244, zweiter Theil Bd. XXIV, S. 217.

14) Note über die Abbildung einer stetigen linearen Mannigfaltigkeit auf eine unstetige, Bd. XXIII, S. 285.

15) Ueber den Inhalt von Punktmengen, Bd. $X X V$, S. 421.

16) Bemerkungen zur Theorie des Doppelintegrales, Bd. XXVI, S. 566.

17) Ueber die mit Ecken behafteten Schwingungen gespannter Saiten, Bd. XXIX, S. 486.

18) Ueber Cauchy's zweiten Beweis für die Convergenz der Fourier'schen Reihe und eine damit verwandte ältere Methode von Poisson. Bd. XXXII. 
II.

In den Sitzungsberichten der physical.-medicinischen Societät zu Erlangen.

1) Ueber die Verwerthung der elliptischen Functionen für die Geometrie der Curven dritter Ordnung, 13. Juli 1874.

2) Zur Theorie der cubischen ternären Formen, 8. Februar 1875.

3) Ueber einen Beweis des Abel'schen Theorems, 12. Juli 1875.

III.

In den Berichten der math. phys. Classe der k. Sächs. Gesellschaft der Wissenschaften zu Leipzig.

1) Beiträge zur Theorie des Cauchy'schen Integrales, 12.Nov. 1885.

2) Existenzbeweise zur Theorie des Potentiales in der Ebene und im Raume, 2. Mai 1886.

3) Ueber die Darstellung einer willkürlichen Function durch die Fourier-Bessel'schen Functionen, 12. Dec. 1887.

IV.

In Schlömilch's Zeitschrift für Mathematik und Physik.

1) Ueber lineare Constructionen von ebenen Curven dritter Ordnung, XXII, S. 38.

2) Zur Theorie der Wärmeleitung in festen Körpern, XXXII, S. 91. $\nabla$.

In anderen Zeitschriften.

1) Ueber algebraische Differentiale, Annali di Matematica, Ser. II, t. 9 , S. 302 .

2) Théorie de la série de Fourier, Darboux, Bulletin des sciences mathém. et astron., ser. II, t. VI, 1883; auch separat erschienen, Paris, Gauthier-Villars 1883.

3) Ueber die einfachsten Methoden zur angenäherten Berechnung ebener Flächen, Civilingenieur, Bd. XXVIII.

VI.

Reden und Festschriften.

1) Ueber den allgemeinen Raumbegriff und seine Anwendbarkeit in der Naturforschung, Sitzungsber. der naturw. Gesellschaft Isis zu Dresden, 1878.

2) Zur Theorie der Wärmeleitung in festen Körpern, Festschrift der Gesellschaft Isis zu Dresden, 14. Mai 1885; vgl. IV, Nr. 2. 
3) Naturforschung und Naturphilosophie, Vortrag gehalten in der naturw. Gesellschaft zu Dresden, Leipzig 1885, Teubner.

4) Festschrift zur Feier des siebenzigsten Geburtstages ihres Vaters Theodosius Harnack, Dr. und Prof. emer. der Theologie an der Universität Dorpat, 3. Jan. 1887; von Dr. A. Harnack, Prof. der Theologie a. d. Universität Marburg, Dr. A. Harnack, Prof. der Mathematik am Polytechnicum zu Dresden, Dr. E. Harnack, Prof. der Medicin a. d. Universität Halle und Dr. O. Harnack, Oberlehrer am Gymnasium Birkenruh, Dresden 1887, Teubner; Ueber den Gebrauch des Unendlichen in der Mathematik, S. 17-29.

5) Leibniz' Bedeutung in der Geschichte der Mathematik. Rede zur Feier des Geburtstages S. M. des Königs von Sachsen, gehalten in der Aula des Polytechnicums zu Dresden, Dresden 1887; Zahn und Jaensch.

\section{VII.}

\section{Recensionen, Referate.}

1) Besprechung des Handbuches der Mathematik, herausgegeben von Schlömilch, unter Mitwirkung von Heger und Reidt, Breslau 1879, 1881; Civilingenieur, Bd. XXIX.

2) Referate über Arbeiten deutscher Mathematiker in Darboux' Bulletin des sciences math. et astron. aus den Jahren 1876-86.

3) Eine Reihe seit 1882 in der allg. Encyclopädie von Ersch und Gruber erschienener zum Theil ausführlicherer Artikel, so z. B. Kettenbruch, Krümmung, Kreis (Beweis der Unmöglichkeit der Quadratur nach Lindemann), Mathematik u. a. m.

\section{VIII.}

\section{Selbständig ersehienene Werke.}

1) Elemente der Differential- und Integralrechnung, 1 Band, $400 \mathrm{~S}$., Leipzig 1881, Teubner.

2) Lehrbuch der Differential- und Integralrechnung von J. A. Serret, mit Genehmigung des Verfassers deutsch bearbeitet von A. Harnack, 3 Bände, Leipzig 1884-85, Teubner.

3) Die Grundlagen der Theorie des logarithmischen Potentials und der Potentialfunctionen in der Ebene, 1 Band, 158 S. Leipzig 1887, Teubner.

Hankel, die Elemente der projectivischen Geometrie in synthetischer Behardlung, herausgegeben von A..Harnack, Leipzig 1875, Teubner. 